\title{
Incentivos fiscales y planificación fiscal de la empresa familiar española: un estudio empírico
}

\author{
Tax incentives and tax planning in Spanish familiy firms: an \\ empirical study.
}

\author{
María José Portillo Navarro $^{\mathrm{a}}$ · Gregorio Sánchez Marín ${ }^{\mathrm{b}, *}$ • José J. García Clavel ${ }^{\mathrm{c}}$ \\ ${ }^{a}$ Departamento de Hacienda y Economía del Sector Público. Universidad de Murcia, 30100 (Spain) \\ ${ }^{b}$ Departamento de Organización de Empresas y Finanzas. Universidad de Murcia, 30100 (Spain) \\ ${ }^{c}$ Departamento de Métodos Cuantitativos para la Economía y la Empresa. Universidad de Murcia, 30100 (Spain)
}

D A T O S A R T Í C U L O

Historial:

Recibido 07-11-2012

Aceptado 23-11-2012

Palabras clave: Incentivos fiscales Planificación fiscal Empresa Familiar Familiness

Agencia

Códigos JEL: M10

\section{A R T I C LE IN F O}

Article history:

Received 07-11-2012

Accepted 23-11-2012

Keywords:

Tax incentives

Tax planning

Family Business

Familiness

JEL codes:

M10

\section{R E S U M EN}

La planificación fiscal de la empresa familiar es imprescindible para garantizar la supervivencia de la misma y poder hacer frente a los costes derivados de los impuestos. Sin embargo, la evidencia nos indica que las empresas familiares están más preocupadas por otros factores de éxito, tales como las relaciones familiares en el seno empresarial. En este trabajo, analizamos las relaciones existentes entre el establecimiento de incentivos del sistema fiscal y la planificación fiscal de la empresa familiar desde la perspectiva del familiness. Sobre la base de una muestra de 282 empresas familiares del sector industrial, se estudia la consistencia de las relaciones entre las dimensiones poder, experiencia y cultura familiar con la capacidad para planificar fiscalmente de las empresas familiares. Los resultados indican que, en general, las empresas familiares están interesadas por una adecuada planificación fiscal cuando se establecen incentivos fiscales que puedan considerar de interés para la adecuada gestión de la empresa familiar.

\section{A B S T R A C T}

Tax planning for family firms is essential to ensure survival through and adequate tax costs managing. However, the evidence indicates that family firms are more concerned about other success factors, such as family relationships within business. In this paper, we analyze the relationship between tax incentives and tax planning for family firms from a familiness perspective. Based on a sample of 282 family firms in the industrial sector, we study the consistency of the relationship between power, experience and culture dimensions of family firms and their ability to plan fiscally. The results indicate that, in general, family firms are more concerned with tax planning when establishing tax incentives that may be of interest for the proper management of the family business.

\footnotetext{
*

Autor de contacto.

Correo electrónico: gresanma@um.es
}

Este trabajo se enmarca en el Proyecto de Investigación CSO2010-17761 del Ministerio de Ciencia e Innovación, "El éxito de la empresa familiar: la relación entre el negocio y la familia desde la perspectiva del familiness". 


\section{Introducción}

La necesidad de una planificación fiscal por parte de todas las empresas resulta evidente, si bien para las empresas familiares es incluso más importante (Scholes et al., 2009), pues aspectos tales como la sucesión o las operaciones vinculadas implican la deseabilidad de una adecuada estrategia a corto $\mathrm{y}$, especialmente, a largo plazo. El carácter de la empresa familiar puede tener incidencia en los incentivos fiscales que utilizan para la minimización de su carga tributaria. Especialmente, la distribución de la presencia y propiedad de la familia en la empresa, la situación generacional de la empresa familiar y la cultura de evasión fiscal de la familia puede determinar en gran medida la planificación fiscal de la empresa y determinar sus resultados, ya que los impuestos pueden afectar al crecimiento, la inversión y la supervivencia del negocio (Astrachan y Aronoff, 1995; Wagner, 1993).

Sin embargo, los estudios que se han centrado en estos aspectos (Chen et al., 2008, 2010; Graham y Tucker, 2006) ha revelado que, en términos generales, las empresas familiares acuden en menor medida al establecimiento de una programación fiscal que el resto de empresas, debido principalmente a que están más preocupadas por la repercusión que pueda tener esta actitud sobre el pequeño accionista, habitualmente miembro de la familia propietaria del negocio. A este respecto, Morris et al. (1997) afirman que las buenas relaciones en el ámbito de la empresa familiar son un factor de éxito más importante que la adecuada planificación fiscal, pues la preocupación por la misma puede llevar al propietario familiar a ignorar otras cuestiones fundamentales de la supervivencia de la familia en el negocio relativas a la planificación de la sucesión y de los futuros papeles de la familia en la empresa.

De la totalidad de cuestiones fiscales que pueden afectar a la empresa familiar, la planificación de la sucesión ha sido tradicionalmente el principal objeto de atención en la literatura (Farhi et al., 2005; Cageti et al., 2007; Molly et al., 2010). Sin embargo, poco se ha investigado acerca de la planificación fiscal del negocio y, más concretamente, sobre la necesidad de implantación de incentivos físcales adicionales que faciliten el crecimiento $y$ sostenibilidad de la empresa familiar. Las pocas excepciones (Chen et al., 2008, 2010) parecen indicar que la capacidad para planificar fiscalmente por parte de la empresa familiar está afectada por la competencia y conocimiento fiscal de los directivos de la empresa familiar, que viene muy determinado por quién ejerce la dirección y ostenta la propiedad de la empresa familiar, cuál es la realidad generacional de la misma y cuál es la cultura de evasión fiscal de la familia empresaria.

En este contexto, Astrachan et al. (2002), apoyándose en la teoría de recursos y capacidades (Barney, 1991; Habbershon y Williams, 1999), construyen el concepto de familiness que, basado en el grado de involucración de la familia en el negocio, supone una definición más amplia de empresa familiar (Ayranci, 2010; Jaskiewicz et al., 2005; Klein et al., 2005) y que puede contribuir a una mejor explicación de la capacidad de planificación fiscal de la empresa familiar. El familiness, que abarca la composición de propiedad y dirección de la familia -dimensión poder-, la experiencia transmitida entre generaciones -dimensión experiencia- y la orientación cultural de la empresa con relación a la familia -dimensión cultura-, es capaz de recoger los principales elementos que configuran la idiosincrasia de la empresa familiar (Rutherford et al., 2008). La influencia que cada una de estas dimensiones ejerce sobre el comportamiento fiscal de la empresa familiar puede arrojar luz sobre su capacidad de planificación fiscal, ya que dicha programación implica decisiones sobre la asunción de impuestos por parte de la propiedad, la necesidad de orientación de recursos en el largo plazo y el compromiso con una determinada cultura empresarial.

Así, en este trabajo, partiendo de una muestra de 282 empresas familiares españolas pertenecientes al sector industrial, se pretende aportar un mayor grado de conocimiento con respecto a la capacidad de planificación fiscal de la empresa familiar, utilizando para ello como principal indicador el conocimiento de los 
incentivos fiscales, así como la necesidad de aplicar incentivos adicionales por parte de la empresa familiar y su vinculación con las diferentes dimensiones del familiness.

Las principales aportaciones del mismo tienen varias vertientes. En primer lugar, se analiza la planificación fiscal de la empresa desde el punto de vista del negocio, yendo más allá de las visiones tradicionales (Astrachan y Tutterow, 1996; Wagner, 1993) que se han centrado básicamente en las implicaciones impositivas en el momento de la sucesión (Chen et al., 2010). Asimismo, en este trabajo se adopta una perspectiva de estudio de la planificación fiscal novedosa, ya que se analiza desde el punto de vista del conocimiento (Chen et al., 2008) sobre incentivos fiscales y de las necesidades con respecto a los mismos por parte de la empresa familiar. Finalmente, este trabajo aporta una visión más amplia de empresa familiar, a través del concepto de familiness (Astrachan et al., 2002; Klein et al., 2005), que supone un análisis integral del carácter familiar de la empresa que va más allá de la mera dirección y propiedad familiar, abarcando aspectos generacionales y culturales, que permiten examinar en qué medida el grado de involucración de la familia en el negocio explica el comportamiento fiscal de la empresa familiar.

Así, el trabajo se estructura de la siguiente manera. En primer lugar, se analizan las principales evidencias sobre planificación fiscal en el ámbito de la empresa familiar, para, a continuación, desarrollar el marco teórico y normativo sobre el que se asientan los principales incentivos fiscales. Posteriormente, el desarrollo del concepto de familiness y sus interrelaciones con la planificación fiscal nos van a conducir a la formulación de las hipótesis de investigación. En tercer lugar, se explica la metodología de análisis empírico, incluyendo la explicación de la muestra y el detalle de la medición de variables. Seguidamente se presentan los resultados estadísticos obtenidos que, finalmente, nos conducen a la presentación de las conclusiones que da pie a la discusión pertinente.

\section{Planificación fiscal de la Empresa Familiar}

\subsection{Revisión de la literatura}

La planificación fiscal es una herramienta imprescindible para el buen funcionamiento de las empresas (Scholes et al., 2009) y las familiares no son ajenas a esta cuestión. El carácter de la empresa familiar puede tener incidencia en los incentivos fiscales que utilizan para la minimización de su carga tributaria. Especialmente, la distribución de la presencia y propiedad de la familia en la empresa (Chen et al., 2008), así como la situación generacional determinan esta situación, ya que los impuestos pueden deprimir el crecimiento empresarial, la inversión y la transmisión del negocio (Astrachan y Aronoff, 1995; Wagner, 1993).

Uno de los principios esenciales de la planificación fiscal es el referido al arbitraje entre operaciones sujetas a distinto trato fiscal (Stiglitz, 1985), lo cual supone la opción por una pluralidad de variables que podrían afectar a la tributación empresarial. Estas variables pueden estar relacionadas con la toma de decisiones en el marco de la normativa tributaria, lo cual incide en la llamada agresividad fiscal de la empresa (Dyreng et al., 2008, 2010), definida como la reducción de la base imponible del impuesto sobre sociedades a través de la realización de actividades de planificación fiscal.

Tradicionalmente se han realizado trabajos en los cuales la planificación fiscal se ha estudiado con independencia de los directivos de la empresa (para revisar esta cuestión, por ejemplo, Graham, 2003). Sin embargo, recientemente, diversos autores han analizado vínculos más profundos entre planificación fiscal y características de la empresa. Así, Desai y Dharmapala (2006) analizan las relaciones entre la evasión del impuesto de sociedades, el crecimiento de los incentivos de alto poder para los administradores, y la estructura de gobierno corporativo. Amstrong et al. (2009) examinan esta cuestión desde la perspectiva de las retribuciones del director tributario (tax director) y la reducción del pago de impuestos. Dyreng et al. (2010) evidencian que las 
características de la dirección y propiedad de la empresa son un factor determinante en el nivel de evasión fiscal de la misma con importantes efectos económicos, lo que se puede trasladar al ámbito de la empresa familiar.

En este sentido, estudios como el de Chen et al. (2010) revelan que las empresas familiares acuden en menor medida al establecimiento de una programación fiscal que el resto de empresas, debido principalmente a que están más preocupadas por la repercusión que pueda tener esta actitud sobre el pequeño accionista, así como por la posible sanción que pudiera derivarse y la reputación familiar. En este trabajo, también se analizan las situaciones de evasión fiscal $\mathrm{y}$, partiendo del estudio de Graham y Tucker (2006), se llega a la conclusión que el porcentaje de las empresas familiares involucradas con paraísos fiscales es del 33 por 100 y es menor que el porcentaje de empresas familiares que se tuvo en cuenta para la muestra analizada, el 46 por 100 (Chen et al., 2010).

Esta idea referida a la menor importancia de la planificación fiscal con respecto a otras cuestiones relacionadas con el éxito de la empresa familiar ya había sido desarrollada en el trabajo realizado por Morris et al. (1997), en el cual se afirma que las buenas relaciones en el ámbito de la empresa familiar son un factor de éxito más importante que la adecuada planificación fiscal, pues la preocupación por la misma puede llevar al propietario/gerente familiar a ignorar otras cuestiones fundamentales del negocio. En cualquier caso, para profundizar adecuadamente en todos los ámbitos de la planificación fiscal de la empresa familiar es necesario distinguir entre: a) planificación de la sucesión, b) planificación fiscal personal y c) planificación fiscal de los negocios.

La planificación de la sucesión de la empresa familiar ha sido tradicionalmente principal objeto de mayor atención en la literatura (Farhi et al., 2005; Cagetti et al., 2007; Molly et al., 2010), puesto que los tributos inherentes a la transmisión lucrativa -herencia o donación- han supuesto tradicionalmente dificultades para la continuidad de la mayoría de empresas familiares. Así, mientras que algunos autores han venido defendiendo la necesidad de la supresión del impuesto que grava las herencias para este tipo de entidades (Cageti et al., 2007) -apoyada por los ordenamientos jurídicos de la mayoría de países (COM 2006, 117)-, encontramos opiniones en otros sentidos. Por ejemplo, Farhi et al. (2005) se plantean si deben existir beneficios fiscales en el momento de la transmisión de riqueza desde una perspectiva de la transmisión intergeneracional de bienestar $y$, más concretamente, autores como Molly et al. (2010) afirman -tras la realización de un estudio de una muestra de 152 pequeñas y medianas empresas durante quince años- no encontrar pruebas de que una empresa familiar se vea afectada en su rentabilidad como consecuencia de la sucesión y, por lo tanto, no debe ser vista como un evento negativo en el ciclo de vida de la empresa.

En otro sentido, hay autores como Grossmann y Strulik (2010), que sugieren que el establecimiento de beneficios fiscales a la sucesión supone un coste económico si los herederos no tienen capacidad suficiente para mantener el negocio familiar $\mathrm{y}$, además, interrumpe la posibilidad de entrada de la iniciativa empresarial. Es decir, el establecimiento de incentivos fiscales en aquellos casos en los cuales los descendientes tienen escasas capacidades empresariales puede causar un efecto perverso, en tanto que los beneficios fiscales pueden animarlos a continuar con la actividad empresarial y hubiera sido mejor que la abandonaran.

La segunda cuestión que es importante distinguir es la relativa a la planificación fiscal personal (Morris et al., 1997). Concretamente, la fiscalidad patrimonial incide negativamente en la eficacia y eficiencia de la empresa familiar que ve reducida su capacidad de autofinanciación (David, 2006; Astrachan y Tutterow, 1996). Los problemas inherentes al Impuesto sobre el Patrimonio han supuesto que muchos países europeos hayan procedido a la supresión del mismo o a la exención de los bienes afectos a la actividad económica.

La tercera cuestión que se plantea relativa a la empresa familiar y la fiscalidad es la referida 
a la planificación fiscal del negocio (Morris et al., 1997). En este sentido, las empresas familiares se enfrentan a cuestiones tales como la relativa a los precios de transferencia, entendidos como los precios a los que una empresa transfiere bienes o presta servicios a empresas con las cuales tiene algún grado de vinculación. A pesar de algunos intentos de creación de bases imponibles consolidadas comunes del Impuesto sobre Sociedades (COM 2011), la mayoría de los ordenamientos aplican el principio de arm's lenght o de plena competencia, por lo que las empresas familiares se ven totalmente afectadas por la normativa tributaria relativa a esta cuestión.

En definitiva, la literatura parece indicar que la planificación fiscal de la empresa familiar está condicionada, entre otros factores, por una cuestión que afecta a la competencia $\mathrm{y}$ conocimiento fiscal de los directivos de la empresa familiar (Chen et al., 2008), que viene muy determinado por quién ejerce la dirección y ostenta la propiedad de la empresa familiar, cuál es la realidad generacional de la misma y cuál es la cultura de evasión/elusión fiscal de la familia empresaria. Por ello, resulta de interés, con el objetivo de conocer la capacidad de planificación fiscal, profundizar en una de las partes de la planificación fiscal más directamente relacionada con el conocimiento y profesionalidad y cultura de la empresa familiar: el reconocimiento de los incentivos fiscales dentro del sistema impositivo a este tipo de entidades y la petición de incentivos adicionales.

\subsection{Incentivos fiscales y planificación fiscal en la empresa familiar}

Una cuestión esencial para el establecimiento de una adecuada planificación fiscal en la empresa es el conocimiento de los incentivos fiscales existentes. En este sentido, el estudio de la necesidad o no de establecer beneficios fiscales adicionales a las empresas familiares que puedan suponer una ayuda al desarrollo de sus actividades para mejorar el beneficio empresarial (Wagner, 1993) y, a la vez, estimular la realización de determinadas acciones que pueden resultar de interés general (Astrachan y Tutterow, 1996), puede ser ilustrativo de los conocimientos fiscales que exhibe la empresa familiar y, por tanto, de su capacidad para realizar una planificación fiscal efectiva.

Puesto que los estudios hasta ahora realizados muestran que las empresa familiares están menos preocupadas que el resto por una adecuada planificación fiscal (Chen et al., 2008, 2010) y reconocida la importancia de la misma en el beneficio empresarial (Astrachan y Tutterow, 1996; Wagner, 1993), en esta investigación se analiza la planificación fiscal de la empresa familiar en función del conocimiento y la necesidad de incentivos fiscales adicionales a los existentes por parte de la empresa familiar.

La necesidad de establecer incentivos fiscales como instrumentos del desarrollo de la política fiscal de cada país es una cuestión que ha sido comúnmente aceptada por los distintos países. Así por ejemplo, en la Unión Europea se establecen deducciones por incentivos en el Impuesto sobre Sociedades, si bien en los últimos años en algunos países como España, se ha optado por la reducción del tipo impositivo y la desaparición progresiva de las deducciones, aunque se siguen manteniendo algunas. Así, en 2011, Alemania tenía establecidos, con un tipo impositivo del 15 por 100 , incentivos por amortización acelerada; en el Reino Unido, con un tipo impositivo del 26 por 100, regula la deducción por investigación y desarrollo; en Francia, con un tipo del 33,3 por 100, se prevé la deducción por amortización acelerada, por creación de empresas, por investigación, desarrollo e innovación, además de otros incentivos al desarrollo regional.

Por otro lado, también existen en la Unión Europea regímenes fiscales favorables a la exención por reinversión, que son favorables para las empresas familiares, pues a menudo tienden a reinvertir las ganancias en vez de repartir dividendos. Así, en países como Malta, Austria o España se conceden beneficios fiscales en el caso de reinversión de beneficios (Austrian Institute for SME Research, 2008). Otros incentivos, tales como los destinados a la 
formación profesional o a las empresas de reducida dimensión, también pueden contribuir al desarrollo de políticas fiscales de interés para la empresa familiar.

Teniendo en cuenta la casuística fiscal europea y española, se consideran representativos de la capacidad de planificación fiscal los siguientes incentivos: (1) deducciones adicionales en I+D; (2) incentivos fiscales adicionales a la realización de gastos de formación profesional en el caso del empresario y sus familiares más cercanos que trabajen en la empresa; (3) establecimiento del régimen especial de empresas de reducida dimensión a todas las empresas familiares; (4) reducción del tipo de gravamen en el Impuesto sobre Sociedades. La utilización de estos incentivos fiscales para las empresas familiares puede ser un indicativo de una planificación fiscal más adecuada y de mayores posibilidades de beneficios empresariales en un sentido amplio.

Los incentivos fiscales a la $I+D$ existen en la mayoría de los países. En este sentido, la Comisión europea adopto en 2006 una Comunicación (COM 2006, 728) referida a la utilización más eficaz de los incentivos fiscales a la I+D. En efecto, la necesidad de aumentar la inversión en I+D llevó al Consejo Europeo a solicitar que se aproximara al 3 por 100 del PIB como máximo en 2010, del cual el 2 por 100 debería provenir del sector privado. Sin embargo, ya se había puesto de manifiesto (COM, 2003, 226 final/2) que el rendimiento social de la inversión en $\mathrm{I}+\mathrm{D}$ suele ser superior al que obtienen las empresas inversoras, por lo que este fallo del mercado era necesario afrontarlo a través del establecimiento de diferentes incentivos y uno de ellos era el fiscal, estableciéndose como prioridad en la COM (2006, 502 final). Estos incentivos fiscales a la I+D en los distintos estados miembros de la Unión europea se han regulado en cada uno de los países miembros de maneras muy diversas y, en algunas ocasiones, estableciendo restricciones territoriales, lo cual ha sido resuelto a través del Tribunal de Justicia de las Comunidades europeas - como en el caso de Francia (Asunto C-39/04) o el de España (Asunto C-248/06), lo cual obligó a la modificación de la legislación en el Impuesto sobre Sociedades - o bien a través de modificaciones legislativas internas, previa iniciativa de la Comisión.

En cualquier caso, el establecimiento de incentivos fiscales a un colectivo determinado como es el de las empresas familiares debe ajustarse a la norma comunitaria sobre ayudas estatales, sujetas a restricciones impuestas por el Tratado de la Unión Europea. En concreto, la COM (2006, 728) establece que "se considera que un incentivo fiscal a la $\mathrm{I}+\mathrm{D}$ es selectivo, $\mathrm{y}$, por tanto, constituye probablemente una ayuda estatal, cuando se limitan sus beneficiarios potenciales en cuanto al tamaño (p. ej., Pyme), la ubicación o el sector". Sin embargo, el establecimiento de deducciones adicionales a la I+D para las empresas familiares -con independencia de su tamaño, el sector en el que desarrollan la actividad o el lugar en el cual la realizan- en principio, no estaría incluida dentro de las ayudas estatales.

$\mathrm{Si}$ bien la literatura a favor del establecimiento de incentivos fiscales adicionales en el Impuesto sobre Sociedades a las empresas familiares es prácticamente inexistente, sí es cierto que en ocasiones se ha planteado la necesidad de establecer beneficios fiscales para las pequeñas empresas e incluso el establecimiento de un tipo impositivo único y la integración del Impuesto sobre Sociedades con el Impuesto sobre la Renta de las Personas Físicas en lo referido a la obtención de rendimientos de la actividad económica, tal y como exponen a través del desarrollo de un modelo de simulación Knitel y Nelson (2011) partiendo de las iniciativas que pretendía llevar a cabo Estados Unidos (National Commision on Fiscal Responsability and Reform, 2010; Guenther, 2009).

Por otro lado, la reducción de tipos impositivos en el Impuesto sobre Sociedades ha sido un tema recurrente en el ámbito fiscal, pues durante años han existido en diferentes sistemas fiscales, tipos impositivos elevados y numerosos incentivos fiscales, por lo que el tipo efectivo disminuía considerablemente. Sin embargo, era una barrera con la cual se encontraban los países, pues las empresas podrían optar por 
deslocalizar el beneficio en aquellos lugares en los cuales el tipo impositivo fuera menor. Por ello, en los últimos años, se ha optado por una reducción de tipos impositivos en numerosos países de la Unión europea, a la vez que se anulaban determinados incentivos fiscales. Así, en 2011, el tipo impositivo general del Impuesto sobre Sociedades de Francia era del 33.33 por 100, de Alemania el 15 por 100, de Italia el 27,5 por 100 , de Irlanda el 12,5 por 100 o de España el 30 por 100, si bien existen tipos especiales para determinados contribuyentes.

Esta situación sería más homogénea si se hubiera conseguido dar pasos más firmes en la armonización fiscal del Impuesto sobre Sociedades. Este deseo de una mayor armonización fiscal, iniciado hace ya varias décadas (ya el Informe Ruding, en 1992, planteaba la necesidad de establecer normas comunes para la determinación de la base imponible y el avance hacia la fijación de tipos mínimos y máximos), no se ha podido materializar como hubiera sido deseable, principalmente debido a la pérdida de soberanía fiscal que puede representar la armonización en este tributo. Los pasos más decididos se han llevado a cabo principalmente a través de la definición de una base imponible consolidada común, tal y como hemos comentado con anterioridad. Pero, en la actualidad, no se plantea la posibilidad de armonizar los tipos impositivos como en un principio se defendía.

En definitiva, el adecuado conocimiento de los incentivos fiscales existentes y el interés por la aplicación de incentivos adicionales por parte de la empresa familiar, viene a ser un indicador representativo de su capacidad de planificación fiscal. Si dicho conocimiento está asociado a factores relacionados con el carácter familiar de la empresa -la dirección y la propiedad familiar, la generación al frente de la empresa y la cultura fiscal de la familiar empresaria-, podemos considerar que el concepto de familiness, basado en el grado de involucración de la familia en el negocio (Astrachan et al., 2002; Klein et al., 2005), es el que mejor recoge los vínculos entre planificación fiscal y empresa familiar. Dichas relaciones se analizan a continuación.

\section{Planificación Fiscal y Familiness}

La teoría de recursos y capacidades de la empresa (Barney, 1991; Makadok, 2003) es la base teórica en la que se fundamenta el concepto de familiness. Esta teoría sugiere que la combinación única de recursos y capacidades de la empresa es su principal fuente de ventaja competitiva (Habbershon y Williams, 1999). Las empresas familiares pueden verse como combinaciones únicas de dos tipos de reglas, valores y expectativas: las de la familia y las del negocio (Gersick et al., 1997). Las empresas familiares comparten ciertas características que las hacen ser únicas en términos de patrones de propiedad, gobierno y generación (Chua et al., 1999; Steier, 2003).

La evolución de una empresa asociada a un único conjunto de condiciones genera recursos imperfectamente imitables, tales como los valores de la familia con relación a la cultura de la organización, el conjunto histórico de activos, o la reputación de la empresa. El conjunto de recursos socialmente complejos de la empresa familiar está profundamente imbricado y es una clara consecuencia del proceso formal e informal de gestión y de toma de decisiones en la empresa familiar (Habbershon y Williams, 1999). Por ejemplo, cuando una empresa experimenta la transición generacional, hay un significativo cambio de recursos y capacidades que incluye información y experiencia, estilo de gestión, relaciones entre accionistas que, por supuesto, afecta a los comportamientos con respecto a la internacionalización. En todos los casos, el familiness de la empresa cambia, suponiendo un cambio general en los resultados y expectativas de la empresa.

El término familiness puede ser descrito, por tanto, como la idiosincrasia de la empresa en el conjunto de sus recursos y capacidades resultantes de la interacción de los sistemas familia-empresa. (Habbershon et al., 2003). En última instancia, Chrisman et al. (2005) expresan el concepto como los recursos y capacidades relacionados con la involucración de la familia en la empresa y sus interacciones. Stafford et al. (1999) toman esta idea y van un paso más allá, integrando los sistemas familiares y de negocio en el mismo nivel que el resto de 
recursos y capacidades de la empresa. Esta aproximación, basada en la creencia de que la involucración de la familia en la empresa puede producir comportamientos que resulten más o menos difíciles de imitar, tiene cabida natural en los planteamientos de la teoría de recursos y capacidades: las empresas familiares existen debido al recíproco valor económico y no económico creado a través de la combinación de los sistemas familiar y de negocio. Además, la intención de mantener la sostenibilidad de la empresa a lo largo de las generaciones puede conducir a la institucionalización del valor percibido de la combinación de los sistemas familiar y de negocio, sugiriendo que la cooperación entre sistemas puede crear utilidades para miembros de la empresa familiar $\mathrm{y}$ adecuar sus comportamientos y decisiones (Rutherford et al., 2008).

De esta forma, el familiness ha sido propuesto, por un lado, como fuente de ventaja competitiva, generador de riqueza y creador de valor para la empresa y, por otro, como un factor de influencia significativa en la gestión de la empresa familiar (Pearson, Carr y Shaw, 2008). De acuerdo a esta segunda noción, Astrachan et al. (2002) propone que el grado en que una empresa es considerada un negocio familiar viene determinado por el modo en que se produce la involucración de la familia definido a través del poder, la experiencia y la cultura- en el negocio. Estas tres dimensiones se adecuan a la escala F-PEC, un índice de la influencia familiar en el negocio, que permite realizar comparaciones entre distintas empresas con diferentes niveles de involucración de la familia, así como analizar los efectos que puede tener en la orientación de determinadas políticas empresariales (Klein et al., 2005).

Es fundamental, por tanto, considerar el contenido global del concepto de familiness para evaluar adecuadamente la capacidad de planificación fiscal de la empresa familiar. El papel específico que la naturaleza del negocio familiar juega, así como la idiosincrasia de este tipo de negocios, hace posible la adopción de diferentes políticas de planificación fiscal según la variación del familiness (Rutherford et al., 2008). Dos empresas con la misma involucración de la familia en términos de gobierno tradicional (propiedad y dirección familiar) podría no presentar la misma orientación fiscal si hay ausencia de intenciones, visión y/o comportamientos orientados a tal actividad y que constituyan la esencia de la empresa familiar.

La dimensión poder se refiere al dominio ejercido a través de la financiación del negocio (por ejemplo, acciones en poder de la familia) y mediante el control del negocio vía participación de la familia en la dirección o el gobierno de la empresa. Una familia puede influir en un negocio vía la extensión y calidad de su propiedad, gobierno e implicación en la gestión (Astrachan et al., 2002; Klein et al., 2005). Esta dimensión hace referencia al grado de influencia genérica o dominancia, ya sea por miembros de la familia propietaria o por directivos a quien ésta designa. Además, estos autores señalan que el nivel de influencia vía poder es aditivo, por lo que cuanto mayor sea el porcentaje de miembros de la familia en cada una de estas funciones (o sin ser miembros de la familia, que hayan sido nombrados por ésta), mayor será la influencia en términos del poder de la familia sobre la empresa.

A este respecto, Chen et al. (2010), utilizando múltiples medidas para captar la agresividad fiscal de la empresa, encuentran que las empresas de propiedad y dirección familiar (de mayor carácter familiar desde el punto de vista del poder) son menos impuestos agresivas fiscalmente que las empresas no familiares, ceteris paribus. Este resultado sugiere que los propietarios mayoritarios de las empresas familiares están dispuestos a renunciar a los beneficios fiscales -incluidos los incentivos adicionales- para evitar el coste que podría suponerles el no pagar impuestos a los ojos de otros accionistas familiares minoritarios, que podrían ver en estas medidas una forma de enmascarar evasiones de impuestos en términos de apropiación indebida de rentas por parte de los mayoritarios (Desai y Dharmapala, 2006).

Este resultado también es coherente con la idea de que las familias propietarias están más preocupadas de los posibles daños legales y reputacionales de una posible auditoría fiscal 
que las empresas no familiares. Esto les lleva a ser menos agresivas fiscalmente y, por tanto, a no asumir riesgos en relación con la búsqueda de deducciones fiscales adicionales. Chen et al. (2010) encuentra conclusiones similares cuando analizan una pequeña muestra de empresas familiares y no familiares en relación con la evasión fiscal: las empresas familiares utilizan en menor medida la evasión fiscal. Estos argumentos nos llevan a la primera hipótesis.

H1: La influencia de la dimensión poder del familiness reduce (tiene un efecto negativo en) la capacidad de planificación fiscal de la empresa familiar.

La dimensión experiencia se refiere a la experiencia conjunta que la familia aporta al negocio a través de la generación o generaciones que activamente participan o han participado en la empresa (Klein et al., 2005). Como argumenta Astrachan et al. (2002), el nivel de experiencia que se produce en la sucesión generacional alcanza su máximo en el cambio entre la primera y segunda generación. La segunda y siguientes generaciones de la propiedad añaden proporcionalmente menos valor a este proceso. Por tanto, la generación de propietarios tiene una relación no lineal con el carácter familiar.

La evidencia empírica, sin embargo, ha sido relativamente distinta en este aspecto cuando se trata de aspectos tributarios, sobre todo como consecuencia de la planificación de la sucesión y sus consecuencias impositivas. Diversos estudios (Farhi et al., 2005; Cageti et al., 2007; Molly et al., 2010) coinciden en afirmar que la no planificación de la sucesión puede acarrear graves consecuencias para la empresa familiar, por lo que las familias suelen estar más preparadas financiera y fiscalmente. Según Klein (2003) a medida que transcurren generaciones, puesto que dicho acontecimiento supone un esfuerzo cada vez mayor por asegurar la continuidad de negocio en manos familiares, es importante contar con la liquidez necesaria para costear los impuestos derivados de la transmisión, así como para satisfacer los intereses de los accionistas y garantizar su bienestar.
En relación con esta idea, algunos autores (Carlock y Ward, 2001; Kuratko et al., 1994) señalan que la magnitud de las consecuencias que encierran los mecanismos para la transferencia de la propiedad -los impuestos y la eventual pérdida de control- hace que el fundador (el paso de la primera a la segunda generación) perciba el proceso de sucesión como algo más complicado y peligroso para la continuidad de su patrimonio que cuando se trata del traspaso de segundas generaciones a las siguientes. Se puede deducir, por tanto, que las segundas y terceras generaciones son más propensas que los fundadores a empezar a planificar esta sucesión $\mathrm{y}$, por tanto, influyen positivamente sobre la capacidad de planificación fiscal de dicha sucesión.

Así, aunque no existe literatura que haya tratado directamente la relación entre planificación fiscal desde la perspectiva de los incentivos fiscales y la situación generacional de la empresa, desde un contexto amplio, los argumentos indican que la entrada en escena de nuevas generaciones se ve como un elemento positivo para estimular la planificación fiscal. Así, considerando estos argumentos, podemos plantear la siguiente hipótesis.

H2: La influencia de la dimensión experiencia del familiness intensifica (tiene un efecto negativo en) la capacidad de planificación fiscal de la empresa familiar

La dimensión cultura se refiere al conjunto de valores, actitudes y motivaciones que imperan en la empresa, y en qué medida se perciben como una conjunción del negocio y de la familia en términos de compromiso con la empresa. En una empresa familiar, la cultura está formada por aquellos valores más enraizados en la organización. Los valores muestran aquello que la familia y la empresa consideran como importante. Según Astrachan et al. (2002) la cultura constituye el área de intersección entre la familia y el negocio que determina que aquellas familias muy comprometidas en el negocio tengan un efecto positivo en la evolución de la empresa (Klein et al., 2005). 
Las empresas familiares con éxito tienden a tener los valores de la familia incorporados a sus estrategias de negocio, políticas y prácticas (Ward, 1998). La construcción de valores compartidos en el seno de familia aumenta la posibilidad del consenso en la toma de decisiones del negocio $\mathrm{y}$, por tanto, facilita el desarrollo de actividades de planificación fiscal.

Kontinen y Ojala (2010) indican que entre las ventajas específicas de las empresas familiares más competitivas e internacionalizadas se incluye un nivel elevado de confianza, valores fuertes y compromiso. El compromiso a largo plazo de las empresas familiares puede ayudar a garantizar a los socios potenciales y a los inversores la continuidad del proceso y un alto nivel de confianza dentro de la organización puede favorecer la formación de vínculos internos y externos. Además, los directivos de las empresas familiares deberían minimizar el efecto de aquellos elementos que dificultarían su continuidad, tales como una inadecuada o inexistente planificación fiscal.

En resumen, la evidencia empírica concluye que aquellas empresas familiares cuyos valores familiares coinciden con la empresa desarrollan una actividad fiscal más intensa y, por tanto, se espera que la dimensión cultura se relacione positivamente con la capacidad de planificación fiscal de la empresa familiar. La hipótesis queda formulada de la siguiente forma.

H3: La influencia de la dimensión cultura del familiness intensifica (tiene un efecto negativo en) la capacidad de planificación fiscal de la empresa familiar.

\section{Metodología}

\subsection{Muestra de empresas y obtención de información}

Para contrastar empíricamente las hipótesis planteadas en la sección previa, se han recogido datos de empresas españolas pequeñas y medianas pertenecientes al sector manufacturero. La población inicial, seleccionada de la base OSIRIS (del Bureau Van Dyck Electronic Publishing), estaba compuesta por un total de 5113 empresas familiares y no familiares de todos los sectores manufactureros con un número de empleados comprendido entre 25 y 249. Sobre esta población se realizó, entre el 28 de febrero y el 31 de marzo de 2011, una encuesta telefónica dirigida al director general de la empresa, obteniendo información válida de 500 empresas (lo que supone un nivel de respuesta del 9,78\%), de las cuales 282 se han autoclasificado como empresas familiares.

\subsection{Medición de variables}

El análisis empírico realizado estudia la relación entre la planificación fiscal y el familiness de las empresas familiares, para lo que se utilizan las siguientes variables.

Capacidad de planificación fiscal. Se ha considerado la capacidad de planificación fiscal de las empresas familiares mediante el conocimiento, necesidad e interés de incorporar beneficios fiscales adicionales al impuesto sobre sociedades. En concreto, se ha preguntado a las empresas por los siguientes beneficios fiscales: (1) deducciones a la formación profesional del empresario y sus familiares más cercanos que trabajen en la empresa; (2) deducciones a la inversión en $\mathrm{I}+\mathrm{D}+\mathrm{i}$; (3) implantación del régimen especial de empresas de reducida dimensión a todas las empresas familiares; y (4) reducción del tipo de gravamen. A partir de aquí se ha construido una variable dicotómica, que toma valores bajo ( $\mathrm{IndFisc}=$ bajo) para aquellas empresas que señalen la necesidad de dos o menos beneficios fiscales y alto (IndFisc $=$ alto) para las que sugieren más de dos.

Empresa familiar. Para medir el carácter familiar de las empresas se ha partido de la escala F-PEC (Astrachan et al. 2002) asociada al concepto de familiness y a las tres dimensiones que lo forman: poder, experiencia y cultura. La dimensión poder se ha medido a través de una variable continua que mide la presencia de la familia en la empresa a través del porcentaje de propiedad de la misma, posteriormente categorizada en dos grupos: $100 \%$ y no- $100 \%$, según esté o no el $100 \%$ de la propiedad en manos de la familia. La dimensión 
experiencia se ha medido mediante la variable generación, que mide la generación que dirige actualmente la compañía y que ha sido categorizada en dos grupos, según se trata de la generación fundadora (primera) $\mathrm{u}$ otra (segunda y siguientes). Finalmente, la dimensión cultura se ha medido a través de 4 ítemes formulados en una escala likert 1-5 (totalmente en desacuerdo - totalmente de acuerdo) considerando si la familia propietaria: (1) tiene influencia en el negocio, (2) comparte valores similares en el negocio, (3) se preocupa por el destino del negocio y (4) sabe diferenciar los aspectos empresariales de los familiares. Los cuatro ítemes fueron sometidos a un análisis factorial secundario obteniendo un solo factor común representativo del constructo, que fue categorizado en tres niveles para el análisis logístico: 1, si la familia influye poco en la cultura de la empresa, 2 si la familia influye moderadamente en la cultura de la empresa, y 3, si la cultura familiar tiene una elevada influencia en el negocio.

\subsection{Análisis estadísticos}

Un primer problema al que nos hemos enfrentado en el análisis estadístico es el número de observaciones de las variables de interés. Aunque el número de empresas encuestadas es de 500, sólo 282 afirman ser empresas familiares, y de ellas sólo 122 afirman conocer beneficios fiscales a través de los cuales hemos medido el concepto de planificación fiscal. Un segundo problema tiene que ver con las medidas de las variables analizadas, todas ellas ordinales y categóricas, dado que las continuas no han ofrecido suficiente variabilidad para ser consideradas como tal (en efecto, para el caso de la dimensión poder, cerca del 101 de las 122 respuestas fueron que el $100 \%$ de la propiedad estaba en manos de la familia).

El método inicialmente propuesto para el estudio era un modelo de ecuaciones estructurales. De hecho, los modelos de medida pudieron ser estimados por separado para cada una de las variables latentes del modelo (poder, experiencia y cultura familiar). Se estudiaron qué indicadores eran los que mejor expresaban los constructos y se hizo un trabajo similar con la planificación fiscal. Sin embargo, a la hora de establecer el modelo estructural que enlazara las diferentes partes del mismo, era necesario -por la naturaleza mixta de las variables empleadas en los diferentes constructos- recurrir a un tipo de correlación específica, la correlación policórica (Joreskog y Sorbom, 1996). Lamentablemente, al menos en el paquete informático que hemos empleado para estimar el modelo (LISREL 8.7) este tipo de correlación utiliza el criterio listwise para tratar los casos perdidos lo que provocaba que nuestra ya escasa muestra se redujera hasta 25 observaciones, lo cual nos invalida la posibilidad de obtención de resultados concluyentes.

Sin embargo, un análisis que resulta adecuado y nos conduce a resultados de gran interés es el expuesto a continuación, pues hemos recurrido a los modelos logarítmicos lineales (MLL) y en concreto la versión logit multinomial (MLM), que permite que una de las variables, en nuestro caso la planificación fiscal, se distinga como variable dependiente dicotómica, mientras que el resto (poder, experiencia y cultura) son las variables independientes cualitativas. De manera simplificada, mediante los modelos logit se obtienen un grupo de parámetros cuya combinación lineal explica las razones esperadas (odds ratio) de la variable dependiente -una explicación detallada de las características de este método puede encontrarse en Agresti (2002) o Greene (2003)-.

\section{Resultados}

Los descriptivos básicos muestran en la tabla 1 las frecuencias cruzadas de las variables de familiness y de planificación fiscal del conjunto de la muestra. Como se puede observar, el conjunto de datos que efectivamente han podido ser cruzados es muy reducido, razón por la cual nos decantamos por el tipo de análisis estadístico anteriormente mencionado.

$\mathrm{Si}$, por ejemplo, observamos las frecuencias de las empresas en las que la familia dirigente es la fundadora, y que tienen un nivel intermedio 
de cultura familiar la razón (odd) de tener una baja preocupación por la fiscalidad respecto a tener una alta preocupación es $2 / 2$, cuando la familia no dispone del $100 \%$ del control y de $1 / 4$ en el caso de que si lo tenga. El cociente entre ambos ratios es de $1 / 0.25=4$, y su logaritmo 1.3863. Si repetimos el mismo cálculo para el caso de que el nivel cultural fuera alto, las cifras serían $3 / 2,20 / 13$ y el cociente entre ambos ratios sería: $60 / 26=2.3077$, cuyo logaritmo es 0.8362 . En ambos casos, el cociente se aleja de la unidad -y por tanto su logaritmo se aleja del cero- por lo que podemos afirmar que la probabilidad de que una empresa familiar tenga una preocupación baja por la planificación fiscal no es indiferente de que el $100 \%$ del control de la misma esté en manos de la familia propietaria. En concreto, es 4 veces más probable que la empresa esté preocupada, para el nivel medio de cultura. Estas diferencias caen, si el nivel cultural de la empresa sube, indicando que se reduce el efecto del poder. Los logaritmos de esos ratios son los parámetros del modelo logit multinomial. Cuanto más cercanos a cero sean, menos significativa será la diferencia entre los niveles de la variable explicativa respecto a la variable dependiente. Un parámetro igual a cero responde a un cociente igual a uno, lo que indicaría que las ratios de los distintos niveles de la variable independiente son iguales.

Para evitar celdas vacías en el modelo, el contraste de hipótesis se ha realizado considerando cada una de las dimensiones del familiness por separado. Así, para la dimensión poder, el análisis logit multinomial, tal y como se observa en la tabla 2 , indica que es más probable que exista una inclinación a sugerir mejoras en la planificación fiscal si el $100 \%$ de la propiedad está en manos de la familia (un $65 \%$ más probable). Lo interesante es el efecto de la interacción, entre planificación fiscal y poder, que toma un valor de -0.585 y es significativamente distinto de cero. Este valor es, como hemos indicado antes, un logaritmo. Transformándolo por su inversa obtenemos 0.5571, es decir, el porcentaje de empresas que tienen un índice de planificación fiscal bajo entre las empresas con un $100 \%$ de la propiedad en manos de la familia es significativamente menor de las que tienen menos del $100 \%$. Por tratarse de parámetros redundantes -por definición la suma de los efectos ha de ser igual a cero, también se puede decir que, entre las empresas con $100 \%$ de la propiedad en manos de la familia, el porcentaje de empresas con una

Tabla 1

Frecuencias cruzadas de las variables analizadas.

\begin{tabular}{|c|c|c|c|c|c|c|}
\hline \multirow{2}{*}{ Cultura } & \multirow{2}{*}{\multicolumn{2}{|c|}{ Experiencia }} & & \multicolumn{2}{|c|}{ Poder } & \multirow{2}{*}{ Tota } \\
\hline & & & & No $100 \%$ & $100 \%$ & \\
\hline \multirow{6}{*}{ Bajo } & \multirow{3}{*}{ Fundadora } & \multirow{2}{*}{ Capacidad de planificación fiscal } & Baja & 1 & 5 & 6 \\
\hline & & & Alta & 0 & 1 & 1 \\
\hline & & \multirow{3}{*}{ Capacidad de planificación fiscal } & & 1 & 6 & 7 \\
\hline & \multirow{3}{*}{ Otra } & & Baja & 3 & 1 & 4 \\
\hline & & & Alta & 2 & 3 & 5 \\
\hline & & Total & & 5 & 4 & 9 \\
\hline \multirow{6}{*}{ Medio } & \multirow{3}{*}{ Fundadora } & \multirow{2}{*}{ Capacidad de planificación fiscal } & Baja & 2 & 1 & 3 \\
\hline & & & Alta & 2 & 4 & 6 \\
\hline & & \multirow{3}{*}{ Capacidad de planificación fiscal } & & 4 & 5 & 9 \\
\hline & \multirow{3}{*}{ Otra } & & Baja & 0 & 3 & 3 \\
\hline & & & Alta & 2 & 9 & 11 \\
\hline & & Total & & 2 & 12 & 14 \\
\hline \multirow{6}{*}{ Alto } & \multirow{3}{*}{ Fundadora } & \multirow{2}{*}{ Capacidad de planificación fiscal } & Baja & 3 & 13 & 16 \\
\hline & & & Alta & 2 & 20 & 22 \\
\hline & & Total & & 5 & 33 & 38 \\
\hline & \multirow{3}{*}{ Otra } & \multirow{2}{*}{ Capacidad de planificación fiscal } & Baja & 0 & 13 & 13 \\
\hline & & & Alta & 4 & 28 & 32 \\
\hline & & Total & & 4 & 41 & 45 \\
\hline
\end{tabular}


Tabla 2

$\underline{\text { Modelo logit multinomial para planificación fiscal y dimensión poder }}{ }^{\mathrm{c}}$

\begin{tabular}{|c|c|c|c|c|c|c|}
\hline \multirow[b]{2}{*}{ Parámetro } & \multirow[b]{2}{*}{ Estimación } & \multirow{2}{*}{$\begin{array}{l}\text { Error } \\
\text { típico }\end{array}$} & \multicolumn{4}{|c|}{$\begin{array}{c}\text { Intervalo de confianza al } \\
95 \%\end{array}$} \\
\hline & & & $\mathrm{Z}$ & Sig. & Límite inferior & Límite superior \\
\hline Constante $\quad[$ poder $=$ no $100 \%]$ & $2,526^{\mathrm{a}}$ & & & & & \\
\hline$[$ poder $=100 \%]$ & $4,182^{\mathrm{a}}$ & & & & & \\
\hline $\begin{array}{l}{[\text { IndFisc }=\text { bajo }] *[\text { poder }=} \\
\text { no } 100 \%]\end{array}$ & $-0,274$ & 0,43 & $0,638^{-}$ & 0,524 & $-1,118$ & 0,569 \\
\hline$[$ IndFisc $=$ bajo $] *[$ poder $=100 \%]$ & $-0,585$ & 0,207 & 2,831 & 0,005 & $-0,99$ & $-0,18$ \\
\hline $\begin{array}{l}{[\text { IndFis } 2=\text { alto }] *[\text { poder }=} \\
\text { no } 100 \%]\end{array}$ & $0^{\mathrm{b}}$ & & . & . & . & \\
\hline$[$ IndFis $2=$ alto $] *[$ poder $=100 \%]$ & $0^{\mathrm{b}}$ & & . & . & . & \\
\hline
\end{tabular}

alta planificación fiscal es significativamente superior. La interacción para el otro caso (el grupo de empresas para las que el poder no está concentrado en una sola familia, las diferencias entre planificación fiscal y bajo poder no es significativamente distinta de cero, como puede verse en la tabla de estimaciones. Por tanto, no podemos aceptar nuestra primera hipótesis, ya que los resultados indican que, en contra de lo esperado, el poder influye positivamente en la disposición a la planificación fiscal.

En la tabla 3 se muestran los resultados del modelo logit multinomial para la dimensión experiencia. En esta ocasión, las diferencias sólo son significativas entre la ratio de los que tienen un alto y un bajo índice de planificación fiscal para el grupo de empresas familiares en las que la generación que dirige no es la primera o fundadura. Y entre ellas, lo que se percibe es que las empresas, lo más probable es que tengan una disposición alta a la planificación fiscal.

También ocurre con las empresas que tienen como dirigente a la generación fundadora pero, en este caso, la diferenciación entre uno y otro grupo no es significativamente distinta de cero (por ejemplo, el intervalo de confianza del parámetro al $95 \%$ va desde $-0,676$ hasta 0,384 , es decir incluye el cero). Por tanto, podemos concluir que la experiencia influye de manera que si la generación no fundadora es la que dirige la empresa, es más probable que se realice una adecuada planificación fiscal, lo que supone aceptar la hipótesis 2.

En el modelo logit multinomial que relaciona planificación fiscal y cultura que presenta la

Tabla 3

Modelo logit multinomial para planificación fiscal y dimensión experiencia ${ }^{\mathrm{c}}$

\begin{tabular}{|c|c|c|c|c|c|c|}
\hline \multirow[b]{2}{*}{ Parámetro } & \multirow[b]{2}{*}{ Estimación } & \multirow[b]{2}{*}{ Error típico } & \multirow[b]{2}{*}{ Z } & \multirow[b]{2}{*}{ Sig. } & \multicolumn{2}{|c|}{ Intervalo de confianza al $95 \%$} \\
\hline & & & & & Límite inferior & Límite superior \\
\hline Constante $\quad[$ exper $=$ Fundadora $]$ & $3,384^{\mathrm{a}}$ & & & & & \\
\hline$[$ exper $=$ Otra $]$ & $3,882^{\mathrm{a}}$ & & & & & \\
\hline$[$ IndFis2 $=$ Bajo $] *$ exper $=$ Fundadora $]$ & $-0,146$ & 0,27 & $-0,539$ & 0,59 & $-0,676$ & 0,384 \\
\hline$[$ IndFis $2=$ Bajo $] *[$ exper $=$ Otra $]$ & $-0,861$ & 0,263 & $-3,269$ & 0,001 & $-1,377$ & $-0,345$ \\
\hline$[$ IndFis $2=$ Alto $] *[$ exper $=$ Fundadora $]$ & $0^{\mathrm{b}}$ & . & $\cdot$ & $\cdot$ & . & . \\
\hline$[$ IndFis $2=$ Alto $] *[$ exper $=$ Otra $]$ & $0^{\mathrm{b}}$ & . & . & . & . & . \\
\hline
\end{tabular}


Tabla 4

Modelo logit multinomial para planificación fiscal y dimensión cultura, ${ }^{\mathrm{d}}$

\begin{tabular}{|c|c|c|c|c|c|c|c|}
\hline \multirow[b]{2}{*}{ Parámetro } & & \multirow[b]{2}{*}{ Estimación } & \multirow[b]{2}{*}{ Error típico } & \multirow[b]{2}{*}{$\mathrm{Z}$} & \multirow[b]{2}{*}{ Sig. } & \multicolumn{2}{|c|}{ Intervalo de confianza al 95\% } \\
\hline & & & & & & Límite inferior & Límite superior \\
\hline \multirow[t]{3}{*}{ Constante } & {$[$ catecul $=1]$} & $1,872^{\mathrm{a}}$ & & & & & \\
\hline & {$[$ catecul $=2]$} & $2,862^{\mathrm{a}}$ & & & & & \\
\hline & {$[$ catecul $=3]$} & $3,998^{\mathrm{a}}$ & & & & & \\
\hline \multicolumn{2}{|c|}{$[$ IndFisc $=$ Bajo $] *[$ catecul $=1]$} & 0,48 & 0,499 & 0,961 & 0,337 & $-0,499$ & 1,458 \\
\hline \multicolumn{2}{|c|}{$[$ IndFisc $=$ Bajo $] *[$ catecul $=2]$} & $-0,99$ & 0,459 & $-2,156$ & 0,031 & $-1,891$ & $-0,09$ \\
\hline \multicolumn{2}{|c|}{$[$ IndFisc $=$ Bajo $] *[$ catecul $=3]$} & $-0,614$ & 0,229 & $-2,685$ & 0,007 & $-1,062$ & $-0,166$ \\
\hline \multicolumn{2}{|c|}{$[$ IndFisc $=$ Bajo $] *[$ catecul $=1]$} & $0^{\mathrm{b}}$ & & . & . & . & . \\
\hline \multicolumn{2}{|c|}{$[$ IndFisc $=$ Bajo $] *[$ catecul $=2]$} & $0^{\mathrm{b}}$ & & . & . & . & . \\
\hline \multicolumn{2}{|c|}{$[$ IndFisc $=$ Bajo $] *[$ catecul $=3]$} & $0^{\mathrm{b}}$ & & . & . & . & . \\
\hline
\end{tabular}

\section{Tabla 5}

Modelo logit para planificación fiscal y dimensiones poder y experiencia

\begin{tabular}{|c|c|c|c|c|c|c|}
\hline \multirow[b]{2}{*}{ Parámetro } & \multirow[b]{2}{*}{ Estimación } & \multirow[b]{2}{*}{ Error típico } & \multirow[b]{2}{*}{ Z } & \multirow[b]{2}{*}{ Sig. } & \multicolumn{2}{|c|}{ Intervalo de confianza al 95\% } \\
\hline & & & & & Límite inferior & Límite superior \\
\hline Constant $[$ poder $=$ no $100 \%] *[$ exper $=\mathrm{Fu}]$ & $1,568^{\mathrm{a}}$ & & & & & \\
\hline$[$ poder $=$ no $100 \%] *[$ exper $=O t]$ & $1,975^{\mathrm{a}}$ & & & & & \\
\hline$[$ poder $=100 \%] *[$ exper $=\mathrm{Fu}]$ & $3,187^{\mathrm{a}}$ & & & & & \\
\hline$[\text { poder }=100 \%]^{*}[$ exper $=\mathrm{Ot}]$ & $3,709^{\mathrm{a}}$ & & & & & \\
\hline$[$ IndFisc $=$ bajo $] *[$ poder $=$ no $100 \%]$ & $-0,641$ & 0,487 & $-1,315$ & 0,188 & $-1,595$ & 0,314 \\
\hline$[$ IndFisc $=$ bajo $] *[$ poder $=100 \%]$ & $-0,923$ & 0,28 & $-3,293$ & 0,001 & $-1,473$ & $-0,374$ \\
\hline$[$ IndFisc $=$ alto $] *$ [poder $=$ no $100 \%]$ & $0^{\mathrm{b}}$ & . & . & . & . & . \\
\hline$[$ IndFisc $=$ alto $] *[$ poder $=100 \%]$ & $0^{\mathrm{b}}$ & . & . & . & . & . \\
\hline$[$ IndFisc $=$ bajo $] *[$ exper $=$ Fundadora $]$ & 0,722 & 0,382 & 1,892 & 0,058 & $-0,026$ & 1,47 \\
\hline$[$ IndFisc $=$ bajo $] *[$ exper $=$ Otra $]$ & $0^{\mathrm{b}}$ & . & . & . & . & . \\
\hline$[$ IndFisc $=$ alto $] *[$ exper $=$ Fundadora $]$ & $0^{\mathrm{b}}$ & . & . & . & . & . \\
\hline$[$ IndFisc $=$ alto $] *[$ exper $=$ Otra $]$ & $0^{\mathrm{b}}$ & . & . & . & . & . \\
\hline
\end{tabular}

tabla 4 resulta interesante comprobar que el único efecto positivo -aunque no significativamente distinto de cero- es para aquellas empresas en las que la familia tiene una presencia muy pequeña en la cultura de la empresa (nivel 1). En este caso, el signo positivo del logaritmo $(0,480)$ revela que la odd ratio es mayor que uno $\mathrm{y}$, por tanto, en este grupo de empresas es más probable que el nivel de conciencia fiscal sea bajo. Para el resto de niveles de cultura, tanto el medio (nivel 2), como el alto (nivel 3), presentan coeficientes 
significativamente diferentes de cero y negativos, lo que indica que el cociente entre las probabilidades es menor que 1 , y por tanto, es más probable que la capacidad de planificación fiscal sea positiva. Así, queda demostrado que la dimensión cultura influye positivamente en la planificación fiscal, pudiendo aceptar la hipótesis 3: cuanto mayor es la presencia de la familia en la cultura de la empresa, más probable es que se deseen incorporar algunos beneficios fiscales.

Finalmente, se han estimado modelos adicionales para comprobar las interrelaciones entre dimensiones de familiness tomadas de forma conjunta y la capacidad de planificación fiscal de la empresa familiar. El tamaño de la muestra no ha permitido combinar todas las variables a la vez, por lo que nos ha llevado a plantear un modelo, con dos de las tres variables explicativas -dimensión poder y dimensión experiencia-. Estas dos dimensiones son muy válidas para representar los resultados conjuntos puesto que representan las dos principales variables sobre las cuales se ha definido tradicionalmente el concepto de empresa familiar.

Como se puede ver en la tabla 5, los resultados confirman que la presencia de la generación fundadora al frente de la empresa familiar hace que sea más probable que la capacidad de planificación fiscal sea baja. Por otro lado, se confirma que si el $100 \%$ de la empresa está controlado por la familia es más probable que la actitud hacia la planificación fiscal sea más positiva. Ambos resultados vienen a confirmar los ya obtenidos en los modelos individuales anteriormente, por lo que dan consistencia a los resultados de este estudio.

\section{Conclusiones y discusión}

En este trabajo analizamos la relación existente entre las diferentes dimensiones del familiness (Astrachan et al.; 2002, Klein et al., 2005) y la planificación fiscal en las empresas familiares. Para ello, partiendo de una muestra de 282 empresas familiares españoles pertenecientes al sector industrial, se analiza la capacidad de planificación fiscal en atención a las preferencias de los empresarios acerca de la posibilidad de aplicar determinados incentivos fiscales en el Impuesto sobre Sociedades, teniendo en cuenta las tres dimensiones que se establecen desde el concepto del familiness: poder, experiencia y cultura. Ello supone la adopción de una perspectiva novedosa y más amplia de empresa familiar que las realizadas hasta el momento.

Si bien la literatura existente señala que las empresas familiares son menos agresivas fiscalmente que las no familiares (Chen et al., 2008, 2010), puesto que están más preocupadas por otros aspectos relacionados con su actividad -tales como las relaciones en el ámbito de la empresa (Morris et al, 1997)- que por el fiscal, la incidencia de una planificación fiscal idónea es muy importante (Sholes et al., 2009), hasta el punto que, en muchas ocasiones, las empresas han desaparecido como consecuencia de una inadecuada planificación fiscal en cuestiones relativas a la sucesión (Fhari et al., 2005; Cagetti et al., 2007; Molly et al., 2010) o a la tributación en el Impuesto sobre Sociedades, pues los impuestos pueden deprimir el crecimiento empresarial, la inversión $\mathrm{y}$, por supuesto, la transmisión del negocio (Astrachan y Aronof, 1995; Wagner, 1993).

Asimismo, el carácter de la empresa familiar puede tener incidencia en los incentivos fiscales que se utilizan para la minimización de la carga tributaria (Chen et al., 2010); en especial si atendemos a un análisis integral del carácter familiar de la empresa definido por el familiness que va más allá de la mera dirección y propiedad familiar, abarcando aspectos generacionales y culturales, partiendo de la teoría de recursos y capacidades de la empresa (Barney, 1991; Makadok, 2003; Habbershon y Williams, 1999; Gersick et al., 1997; Chua et al., 1999; Steier, 2003) que permite examinar en qué medida el grado de involucración de la familia en el negocio explica el comportamiento fiscal de la empresa familiar. En efecto, las características de dirección y propiedad de la empresa son un factor determinante en el nivel de evasión fiscal de la misma (Dyreng et al., 2010) con importantes efectos económicos, lo cual es trasladable a la empresa familiar. 
Si bien en los escasos estudios existentes acerca de la planificación fiscal de la empresa familiar se suele atender a criterios de agresividad fiscal (Chen et al., 2010) o de planificación fiscal que incluyen la evasión y la elusión fiscal, en nuestro trabajo atendemos al concepto de planificación fiscal de Scholes (2009) que implica la reducción de la carga tributaria del contribuyente en atención a la utilización de los incentivos fiscales que establecen las normas tributarias pero siempre en el marco de la ley, sin acudir al incumplimiento de la legislación (evasión) ni al fraude de ley (elusión).

El estudio de la relación entre la planificación fiscal y las dimensiones familiness lo hemos articulado a través del análisis de tres hipótesis de trabajo. En primer lugar, hemos planteado la hipótesis relativa a la existencia de una relación inversa entre las empresas familiares con una mayor dimensión poder y la planificación fiscal. La dimensión poder se refiere al dominio ejercido por la familia en la dirección o gobierno de la empresa, a través, por ejemplo, de acciones propiedad de la familia, puesto que una familia puede influir en el negocio a través de la extensión, calidad de la propiedad, gobierno o implicación en la gestión (Astrachan et al., 2002; Klein et al., 2005). Por lo tanto, cuanto mayor sea el porcentaje de miembros de la familia en la dirección (directamente o a través del nombramiento de directivos) mayor será la influencia en términos de poder de la familia sobre la empresa. La escasa literatura al respecto defiende que las empresas de propiedad y dirección familiar (con mayor carácter familiar desde el punto de vista del poder) son menos agresivas fiscalmente que las no familiares (Chen et al., 2010) y están más preocupadas por lo que podrían pensar otros accionistas minoritarios si realizan actividades de evasión fiscal (Desai y Dharmapala, 2006).

Sin embargo, los resultados obtenidos evidencian que para la dimensión poder, las empresas familiares con un $100 \%$ de la propiedad en manos de la familia realizan una mayor planificación fiscal que las que mantienen una menor proporción de propiedad en sus manos. Por lo tanto, no podemos aceptar nuestra hipótesis inicial, pues el poder influye en una mayor disposición a la planificación fiscal. Estas diferencias con respecto a la literatura mencionada pueden deberse a varios motivos. En primer lugar, tanto Chen et al. (2010) como Desai y Dharmapala (2006) el concepto que utilizan incluye tanto la planificación fiscal en el marco de la ley conducente a minimizar la factura fiscal y, por tanto en el marco legal, como la posibilidad de realizar políticas de evasión y elusión fiscal fuera de la ley. Mientras que, en nuestro caso, utilizamos el concepto de planificación fiscal restringido; es decir, operaciones en el marco de la ley que reducen la carga tributaria del contribuyente si opta por la realización de determinadas políticas incentivadas desde una perspectiva fiscal (Scholes, 2009).

Otra cuestión por la cual nuestros resultados difieren de Chen et al. (2010) es que mientras su análisis compara entre empresas familiares y no familiares, en nuestro caso la muestra comprende solamente empresas familiares, con el consiguiente problema de falta de variabilidad. Efectivamente, se ha comprobado que la gran mayoría de empresas familiares de nuestra muestra presenta una dimensión poder muy similar, ya que la mayor parte de ellas tiene un porcentaje de propiedad y de dirección familiar cercano al $100 \%$, lo que puede haber provocado la falta de resultados significativos.

Por lo tanto, a la vista de los resultados obtenidos, podemos interpretar que cuando la empresa familiar puede incurrir en costes de imagen derivados de la evasión o la elusión fiscal, está menos interesada en la planificación fiscal que en el caso en el que esta programación fiscal pueda suponer la realización de políticas dentro de la empresa que se encuentren incentivadas fiscalmente, como puede ser la inversión en $\mathrm{I}+\mathrm{D}+\mathrm{i}$ o la realización de gastos de formación profesional y no puedan suponer un problema relacionado con la imagen empresarial (Morris et al., 1997).

La segunda hipótesis establece que la dimensión experiencia del familiness intensifica la capacidad de planificación fiscal en la empresa familiar. La dimensión experiencia viene referida a la experiencia conjunta que la 
familia aporta al negocio a través de la generación o generaciones que participan en ella y su mayor preparación para la planificación fiscal y financiera (Farhi et al., 2005; Cagetti, 2007; Molly et al., 2010) y, a medida que transcurren generaciones, según Klein (2003), es imprescindible contar con la liquidez necesaria para poder costear los impuestos y, de ese modo, poder satisfacer los intereses de los accionistas. Tras los resultados obtenidos, podemos afirmar que la hipótesis planteada se cumple, por lo que podemos concluir que la experiencia influye en la planificación fiscal positivamente. Es más, en el caso en el que la empresa familiar cuente con un dirigente de segunda o sucesivas generaciones, esta hipótesis es todavía más consistente que en el caso en el que se trate de una empresa dirigida por el fundador.

Por último, la tercera hipótesis establece que en aquellas empresas en las cuales la influencia de la dimensión cultural del familiness es mayor, son más proclives a una mayor planificación fiscal, existiendo por tanto una relación directa entre ambas variables. La dimensión cultura hace referencia al conjunto de valores, actitudes y motivaciones que imperan en la empresa $\mathrm{y}$, más concretamente en la empresa familiar. La cultura está formada por aquellos valores más enraizados en la organización y que la familia empresaria considera como más importantes (Astrachan et al., 2002), teniendo las empresas familiares de éxito los valores de la familia incorporados a sus estrategias de negocio, política y prácticas (Ward, 1998).

Tras el análisis realizado, se confirma la hipótesis de que la dimensión cultura influye positivamente en la planificación fiscal. Por lo tanto, cuanto mayor es la presencia de la familia en la cultura de la empresa, más probabilidades existen de la consideración de la incorporación de beneficios fiscales. Se confirma claramente pues el efecto relativo a que la alineación de la cultura familiar con la cultura del negocio hace que los directivos familiares estén más preocupados por la planificación estratégica del negocio, lo cual les lleva a ser cuidadosos por plantear una adecuada planificación fiscal
(Desai y Dharmapala, 2006; Amstrong et al., 2009; Dyreng et al., 2010).

En definitiva, podemos concluir que las empresas familiares, cuando se le ofrecen incentivos fiscales que implican la actuación en el marco de la ley, están interesadas en realizar una adecuada planificación fiscal y esta opción se intensifica en el caso de empresas familiares con una mayor dimensión experiencia o cultura en los términos definidos por el familiness. En este sentido, a pesar de que la dimensión poder, relacionada con la propiedad y dirección familiares en la empresa no ha resultado significativa en el sentido esperado, estamos convencidos de que la misma ejerce un efecto importante, pero que debido a las características tan homogéneas de nuestra muestra en relación a dicha dimensión, no han podido ser capturadas de forma adecuada.

En futuros trabajos sería conveniente analizar otras vías de estudio, tales como el interés mostrado por los empresarios familiares por unos u otros instrumentos de planificación fiscal o bien la posibilidad de relacionar las preferencias mostradas con otras variables, tales como ventas, beneficios o inversiones, que puedan resultar indicativas para el establecimiento de nuevas vías de estudio de la presente línea de investigación.

\section{Referencias}

Agresti, A. (2002): Categorical Data Analysis. Wiley-Interscience, New York.

Armstrong, C.S., Blouin, J.L., \& Larcker, D.F. (2009): The incentives for tax planning. Stanford GSB Research Paper No. 2032 and Rock Center for Corporate Governance Working Paper No. 56.

Astrachan, J.H., \& Aronoff, C.E. (1995): A report on the impact of federal estate tax: A study of two industry groups. Family Enterprise Center. Marietta, GA.

Astrachan, J.H., \& Tutterow, R. (1996): The effect of estate taxes on family. Family Business Review, 3, 303-314.

Astrachan, J.H.; Klein, S.B. \& Smyrnios, K.X. (2002): The F-PEC scale of family influence: A proposal for solving the family business definition problem, Family Business Review, 15, 44-58. 
Austrian Institute for SME Research (2008) (ed). Overview of Family Business Relevant Issues. Final Report

Ayranci, E. (2010). Family involvement in and institutionalization of family businesses: A research. Business and Economic Horizons, 3(3), 83-104.

Barney, J.B. (1991). Firm resources and sustained competitive advantage. Journal of Management, 17, 99-120.

Cagetti, M., \& De Nardi, M. (2007): Estate taxation, entrepreneurship, and health. Working Paper 13160. National Bureau of Economic Research.

Carlock, R., \& Ward, J. (2001). Strategic planning for the family business: Parallel planning to unify the family and business. Londres: Palgrave.

Chen, S., Chen, X., \& Cheng, Q. (2008): Do family firms provide more or less voluntary disclosure? Journal of Accounting Research, 46, 499-536.

Chen, S., Chen, X, Cheng, Q, \& Shevlin, T. (2010): Are family firms more tax aggressive than non-family firms? Journal of Financial Economics, 95, 41-61.

Chrisman, J.J., Chua, J.H., \& Sharma, P. (2005). Trends and directions in the development of a strategic management theory of the family firm. Entrepreneurship Theory and Practice, 29, 555575.

Chua, J.H., Chrisman, J.J., \& Sharma, P. (1999). Defining the family business by behavior. Entrepreneurship Theory and Practice, 23, 19-39.

$\operatorname{COM}(2003)$ : Investing in research: an action plan for Europe. Communication from the Commission, 226 final/2, European Union.

COM (2006): Implementing the Lisbon Community Programme for Growth and Jobs. Transfer of Businesses - Continuity through a new beginning. European Commission Communication, 117 final. European Union.

COM(2006): Putting knowledge into practice: A broad-based innovation strategy for the EU. Communication from the Commission to the Council, the European Parliament, the European Economic and Social Committee and the Committee of the Regions, 502 final

COM (2006): Hacia una utilización más eficaz de los incentivos fiscales a la $I+D$. European
Commission Communication, 728 final. European Union

COM (2011): Proposal for a COUNCIL DIRECTIVE on a Common Consolidated Corporate Tax Base (CCCTB). 121/4. European Union

David, J. (2006): The behavioral response of wealth accumulation to estate taxation: time series evidence. National Tax Journal, 59, 253-268.

Desai, M., \& Dharmapala, D. (2006): Corporate tax avoidance and high-powered incentives. Journal of Financial Economics, 79, 145-179.

Dictamen del Comité Económico y Social Europeo (2006): Creación de una base imponible consolidada común para el impuesto de sociedades en la UE. Dictamen 2006/C 88/12, CESE.

Dyreng, S. D., Hanlon, M. \& Maydew, E.L. (2008): Long-run corporate tax avoidance. The Accounting Review, 83, 61-82.

Dyreng, S. D., Hanlon, M. \& Maydew, E.L. (2010): The effects of executives on corporate tax avoidance. The Accounting Review, 85, 1163-1189.

Farhi, E., \& Werning, I. (2005): Inequality, social discounting and estate taxation. Working Paper 11408. National Bureau of Economic Research.

Gersick, K.E., Davis, J.A., McCollom Hampton, M., \& Lansberg, I. (1997). Generation to generation: Life cycles of the family business. Boston, MA: Harvard Business School Press.

Graham, J. (2003): Taxes and corporate finance: A review. Review of Financial Studies, 16, 10741128.

Graham J. R, Tucker A (2006): Tax Shelters and Corporate Debt Policy. Journal of Financial Economics, 81, 563-594.

Greene, W.H. (2003): Econometric Analysis, fifth edition. Prentice Hall, New York.

Grossmann, V., \& Strulik, H. (2010): Should continued family firms face lower taxes than other estates? Journal of Public Economics, 94, 87-101.

Guenther, G. (2009): Small business tax benefits: overview and economic rationale. Congressional Research Service.

Habbershon, T. \& Williams, M. (1999): A resource-based framework for assessing the strategic advantages of family firms. Family Business Review, $12,1-25$.

Habbershon, T., Williams, M., \& MacMillan, I. (2003): A unified systems perspective of family firm 
performance. Journal of Business Venturing, 18, 451-465.

IBFD (ed.) (2011): European tax handbook.

Jaskiewicz, P., Gonzalez, V., Menendez, S., \& Schiereck, D. (2005): Long-run IPO performance analysis of German and Spanish family-owned businesses. Family Business Review, 18, 179-202.

Joreskog, K. Sorbom, D. (1996): LISREL 8: User's Reference Guide. Scientific Software International, Chicago.

Klein, S.B. (2003): Strategic Renewal during Succession in Family Firms: How to benefit from the succession process. In Poutziouris, P., Steier, L.P. (Eds): New frontiers in family business research the leadership challenge. Research Forum Proceedings, pp. 196-215. FBN-IFERA Conference, Lausanne.

Klein, S.B., Astrachan, J.H., \& Smyrnios, K.X. (2005): The F-PEC scale of family influence: Construction, validation, and further implication for theory. Entrepreneurship Theory and Practice, 29, 321-339.

Kontinen, T. \& Ojala, A. (2010): The internationalization of family businesses: A review of extant research. Journal of Family Business Strategy, 1, 97-107.

Kuratko, D., Foss, H., \& Vanalst, L. (1994): IRS estate freeze rules: implications for family business succession planning. Family Business Review, 7, 6171.

Makadok, R. (2003): Doing the right thing and knowing the right thing to do: Why the whole is greater than the sum of the parts. Strategic Management Journal, 24, 1043-1055.

Molly, V., Laveren, E., \& Deloof, M. (2010): Family business succession and its impact on financial structure and performance. Family Business Review 23, 131-147.

Morris, M., Williams, R., Allen, J., \& Avila, R. (1997): Correlates of success in family business transitions. Journal of Business Venturing, 12, 385401

National Commission on Fiscal Responsibility and Reform (2010): The moment of truth. NCFRR.
OCDE (1995): Transfer Pricing Guidelines for Multinational Enterprises and Tax Administrations. OCDE, Paris

Pearson, A.W., Carr, J.C. \& Shaw, J.C. (2008). Toward a theory of familiness: A social capital perspective. Entrepreneurship Theory and Practice, 32, 949-969.

Recomendación de la Comisión (1994): Transmisión de las pequeñas y medianas empresas. Recomendación 94/1069/CE.

Rutherford, M.W., Kuratko, D.F., \& Holt, D.T. (2008): Examining the link between "familiness" and performance: Can the F-PEC untangle the family business theory jungle? Entrepreneurship Theory and Practice, 32, 1089-1109

Scholes, M., Wolfson, M., Erickson, M., Maydew, E. \& Shevlin, T. (2009): Taxes and business strategy: A planning approach. Pearson Prentice Hall. Upper Saddle River, NJ.

Stafford, K., Duncan, K.A., Dane, S., \& Winter, M. (1999): A research model of sustainable family businesses. Family Business Review, 12, 197-208.

Steier, L. (2003). Variants of agency contracts in family financed ventures as a continuum of familial altruistic and market rationalities. Journal of Business Venturing, 18, 597-618.

Stiglitz, J. E. (1985): The general theory of tax avoidance. National Tax Journal, 38, 325-337.

U.S. Department of the Treasury (1992): Integration of the individual and corporate tax systems: taxing business income once. U.S. Government Printing Office, Washington, DC.

Wagner, R. (1993): Federal transfer taxation: A study in social cost. The Center for the Study of Taxation. Costa Mesa, CA.

Ward, J.L. (1998). Growing the family business: special challenges and best practices. Family Business Review, 10, 323-337. 